\title{
Rural Settlements in Different Stages of Development. Case of Gjirokastra Region in South of Albania
}

\author{
Prof. Ass. Albina Sinani \\ Department of Geography, \\ Faculty of Education and Social Sciences, Gjirokastër \\ "Eqrem Çabej" University, Gjirokastër, Albania \\ E-mail: sinanialbina@yahoo.com
}

Doi:10.5901/mjss.2013.v4n2p533

\begin{abstract}
:
Recent years has been put into scientific circulation rich documentation, in which data is important for rural settlements and their economy. First Ottoman cadastral registers, which have a value first hand, but also for a more extensive documentation which comes out more clearly the face of the Albanian medieval village, the size, the main economic resources, relations with civic centers, economic power of the settlements, the role of agriculture in village life, crops etc.. The size of the settlements and their economy were closely related to each other. Most have been populated hilly South Albania and less western coastal lowlands of Albania and hilly villages in their vicinity. Construction of various settlements of stone was spread rapidly for a period of time. Stone materials were present everywhere and therefore their spread was massive and quite fast. Rural areas are characterized by a landscape architecture built by 2-3 storey residential houses. Typology is varied and reflects the special features of natural, historical, psychological, ethnographic and economic. In the construction of rural settlements have been preserved traditional elements, with special features according to the different areas and historic development.
\end{abstract}

Keywords: rural settlements; special features; architectural aspect; buildings; establishment; landscapes;

\section{Introduzione}

Gli insediamenti rurali nel distretto di Girocastro sono mutati nel tempo, in conseguenza al cambiamento dei fattori socioeconomici, al modo di vivere, al modo di edificare, in base all'evolversi delle esigenze degli abitanti e dell' adattamento di questi edifici alle loro attività commerciali. L'architettura di queste costruzioni, tramandata negli anni è variegata e molto particolare. Le costruzioni sono composte in tutti i casi da due aree, quella funzionale e quella ornamentale della casa stessa, come espressione fedele della natura, dell'etnografia, della storia, dell'economia, dell'amministrazione e della psicologia della cultura del territorio ereditata negli anni.

\section{Periodo prima dell'anno 1945}

Le caratteristiche tradizionali, locali e la complessità architettonico - urbanistica dello spazio rurale di Girocastro risale al IV secolo a.c. La loro storia viene caratterizzata da importanti tecniche architettoniche usate nell'edificare le costruzioni. L'evoluzione degli insediamenti rurali negli edifici successivi si basa nella tradizione autoctona e nella professionalità dei maestri costruttori. Facendo un'analisi delle prime costruzioni rurali spicca la loro doppia funzione.

La prima funzione è quella abitativa, dove si praticano le norme sociali, tecniche, folcloristiche ecc.

La seconda funzione consiste nello sfruttare ad usi economici lo spazio interno ed anche quello esterno. La principale caratteristica delle varie funzioni svolte nell'edificio rurale si esprime nelle dimensioni della casa e nel suo ordine interno, dove si recupera spazio per organizzare il raccolto, allevare il bestiame e ordinare gli attrezzi agricoli.

Le case includevano anche una serie di ripostigli uno affianco all'altro e una cantina, un ambiente fresco usato per l'essicazione e l'elaborazione del raccolto agricolo. L'utilizzo funzionale del luogo di costruzione, la varietà dei materiali usati, l'alto livello di elaborazione degli stessi e le tecniche precise nella costruzione delle mura dell'edificio rurale e delle strutture che lo affiancano (casa con forche di legno, adobe, pietra), ne deducono una consolidata tradizione di 
costruzione ed un utilizzo ottimizzato della terra dove si costruisce. Le soluzioni pratiche dei problemi funzionali ed architettonici, hanno fatto si che le costruzioni durassero a lungo nel tempo. Le case rurali fanno da strumento agricolo, perciò le loro dimensioni, la loro forma ed il loro utilizzo all'interno si adatta nella dimensione spazio-temporale ad una tipologia precisa agricola e ad un sistema di colture agricole.

Foto 1. Vecchia casa, Labova e Kryqit (A. Sinani)

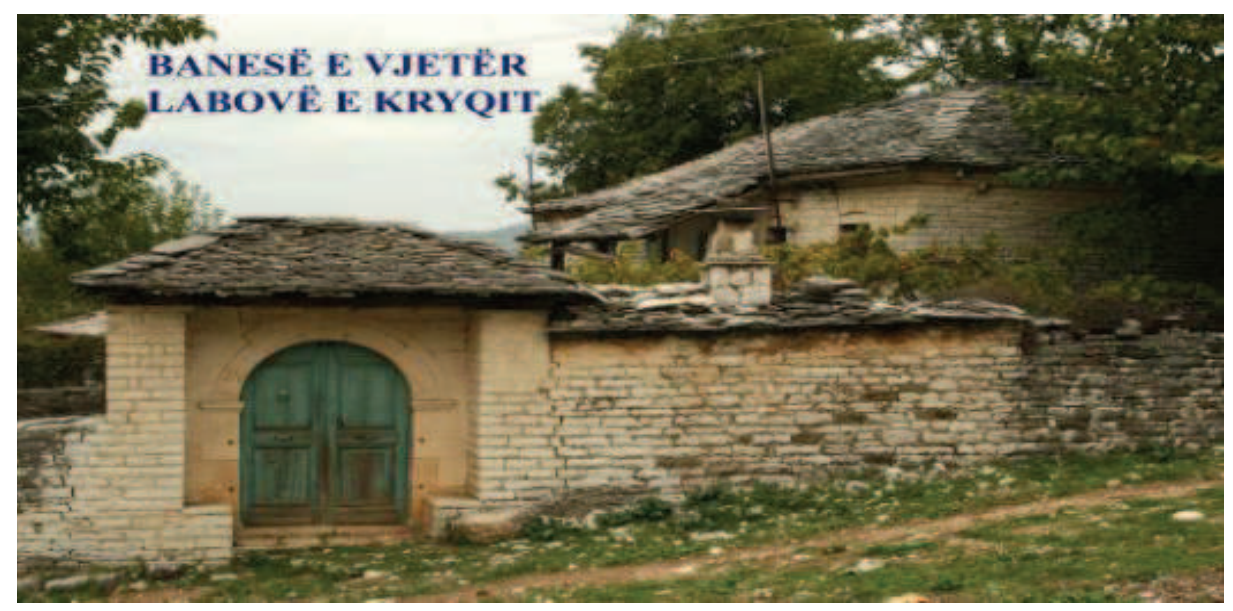

Gli insediamenti rurali in tutta l'area di Girocastro risalgono a molto tempo fa. La maggior parte della popolazione di questi, era concentrata nelle zone di montagna, dove si sopravviveva di un'economia basata sull'allevamento del bestiame (Riza, 1991). La casa era molto estesa e si viveva con una gerarchia patriarcale. Per permettere anche la funzione abitativa di consueto la casa era di due piani. Esternamente davanti alla casa c'era il giardino che lo ritroviamo recintato o meno da un muro di pietre. Per la pavimentazione del primo piano della casa venivano utilizzate delle lastre di pietra ed era quasi sempre presente una scala che usciva nella parte posteriore della casa (la cantina, i luoghi del bestiame), e permetteva di collegare la casa a questi luoghi. Sempre nel primo piano si trovava un deposito dei latticini e delle riserve di cibo, usato d'inverno per le persone e gli animali. II secondo piano svolgeva solo funzioni abitative per i componenti della famiglia e includeva una grande stanza degli ospiti.

Foto 2. Vecchia casa, Lipe (A. Sinani)

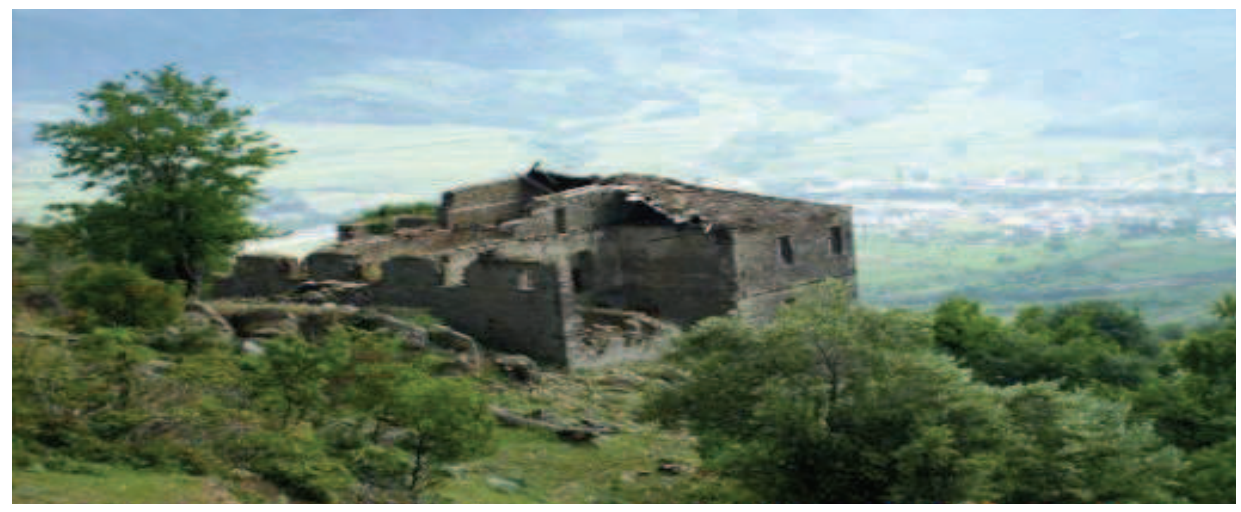

Le case dei contadini modesti erano basse e senza soffitto, in modo che il fumo potesse uscire con più facilità, le mura erano fatte di fango e paglia. Esisteva anche una tipologia di case che univa sotto lo stesso tetto i luoghi abitativi 
con quelli di utilizzo agricolo. Quest'ultime sono costruzioni fatte solo del pian terreno oppure anche di un mezzo piano costruito di pietra. (Thëngjilli, 2004, p. 268).

Le testimonianze dei viaggiatori stranieri verso la fine del XVIII sec. e l'inizio del XIX fanno distinguere due tipologie di abitazioni diverse per le dimensioni e per lo spazio dedicato all'uso agricolo.

Foto 3. Vecchia casa, Kosove (A. Sinani)

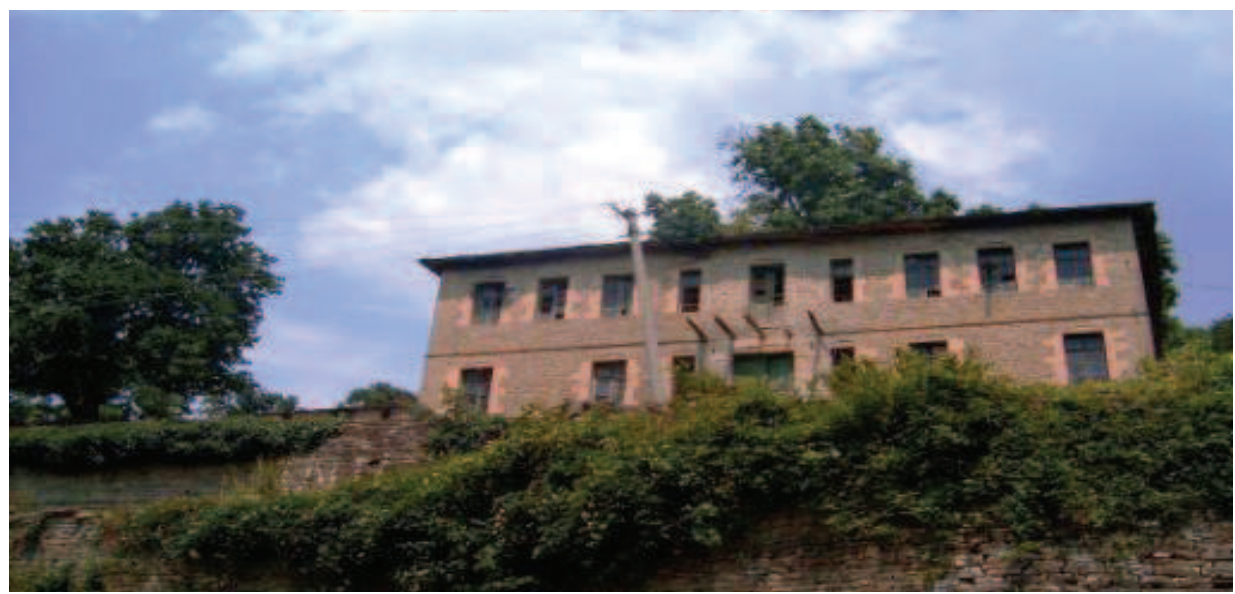

Le abitazioni a forma di torre ed a carattere difensivo. L'apoteosi nella costruzioni di abitazioni fortificate a carattere difensivo giunge nel periodo tra la fine del XVIII sec. e l'inizio del XIX. Gli edifici costruiti erano alti ed avevano una doppia funzione di sorveglianza e di stabilità (Muka, 1990). Le abitazioni-fortezza le ritroviamo principalmente nella valle di Dropull di sotto e nel paese di Goranxi. Solo in questo si trovavano più di 25 fortezze, delle quali 8 ancora esistenti (soltanto 3 allo stato originale come la dimora di Ngjelaj e la dimora di Kamberaj).Come queste abitazioni potremmo citare quelle trovate nei paesi di Haskove, Sofratike, Dervician, Kardhiq, Golem ecc.

Foto 4. Vecchia casa, Kosove (A. Sinani)

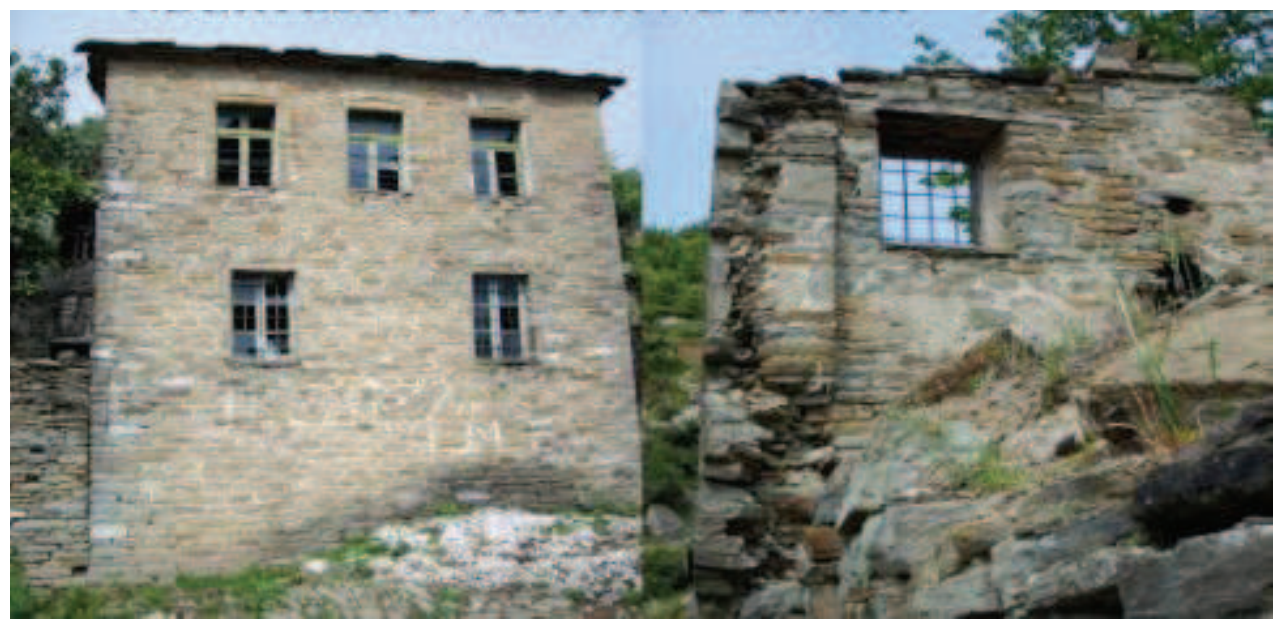

Le abitazioni con costruzioni divise e specializzate. Questa tipologia di abitazioni la ritroviamo in modo molto diffusso nelle vicinanze delle terre fertili, sotto le montagne oppure sulle colline cosi da avere anche una funzione 
difensiva. Le abitazioni rurali hanno fatto da filo conduttore alle abitazioni urbane più perfezionate. D'altro canto quest'ultime hanno fatto si che abitazioni rurali si trasfomassero nel tempo ed evolvessero, distaccandosi dalla tipologia tradizionale. L'organizzazione dello spazio, l'evoluzione rurale e le prospettive dello sviluppo stabile nel distretto di Girocastro.

Foto 5. Gjiokastra casa (A. Sinani)

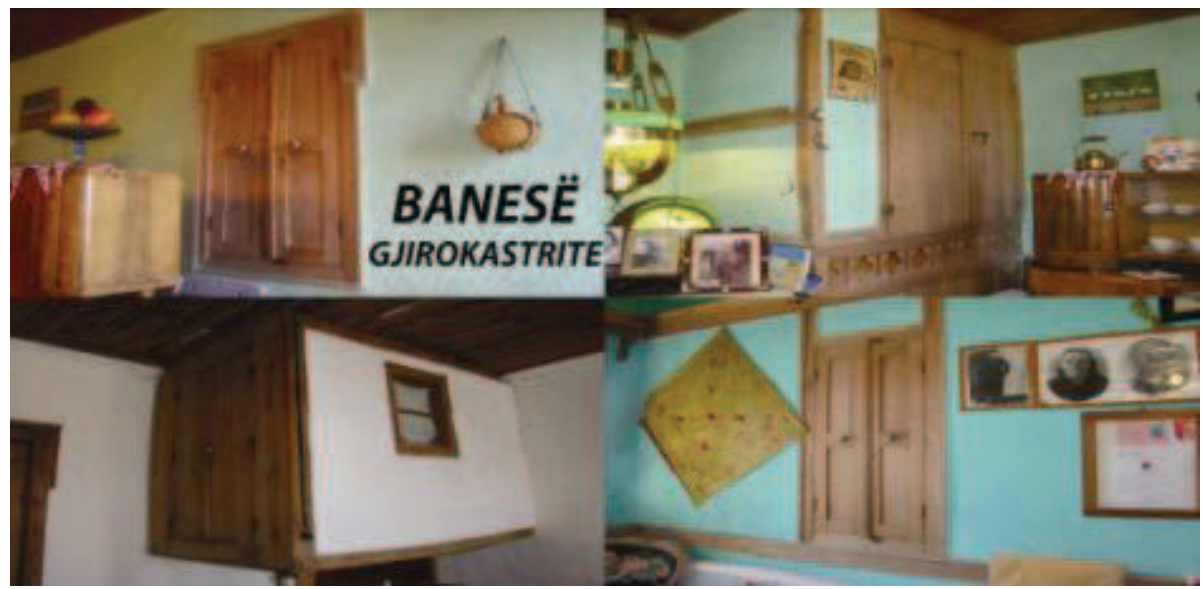

Le testimonianze di tali costruzioni le ritroviamo nelle abitazioni esistenti in questo periodo, ed hanno valori storici importanti. Possiamo menzionare le rovine trovate nel paese di Dardhe, ad est della città di Permet, sulla sponda destra del fiume Vjose. Le rovine delle costruzioni ritrovate completano il quadro delle abitazioni di questo periodo, edificate con tecniche remote, dove veniva sfruttato la collocazione in parallelo delle isopsia, aggiungendo in questo modo importanti valori architettonici e decorativi. Maggior parte delle case si trovavano in zone collinari e le terre fertili si trovavano giù sistemate e delineate, per evitare possibili frane nelle strade del paese. II centro del paese si trovava quasi sempre vicino a delle fonti d'acqua. Le abitazioni tipo torre a due piani icludevano nella costruzione la torretta d'osservazione, elemento fortificante che ha conessione diretta con la situazione sociale, economica e storica del momento. La tecnica dominante era quella di costruzione con mura di pietre e calce su argilla malta ed anche stecche di legno inchiodate con chiodi smith.

Foto 6. Vecchio villagio, Grapsh (A. Sinani)

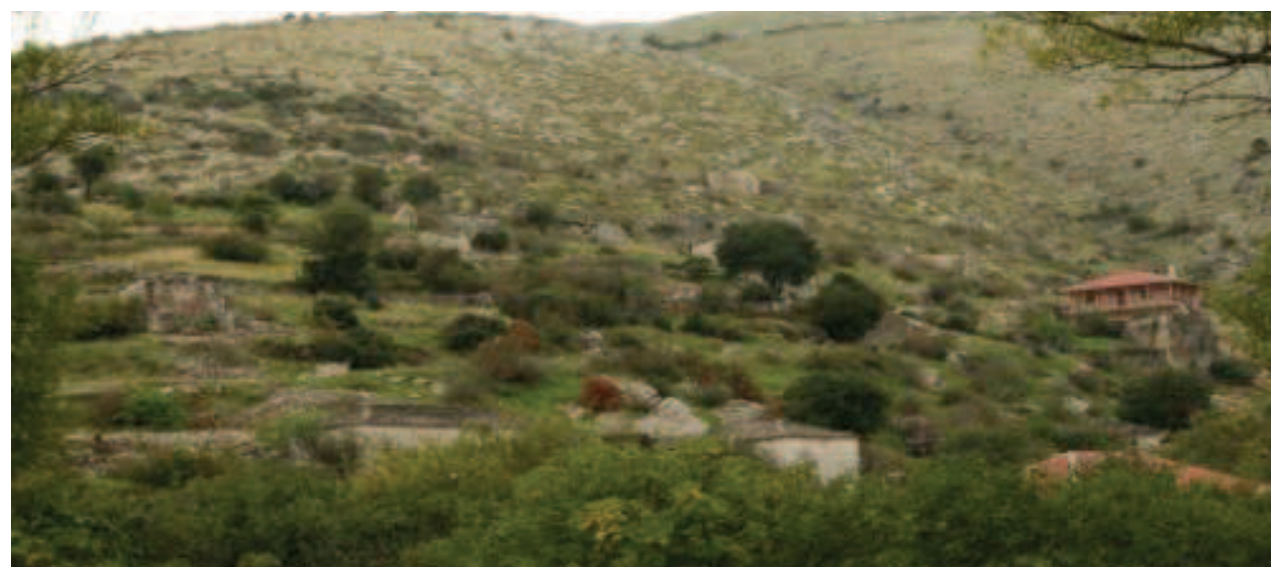


L' abitazione si estendeva in tre livelli di terra, quello medio al interno del paesino, quello superiore, in montagna, che faceva da pascolo estivo e quello inferiore,dove c'erano le terre fertili, che facevano da pascolo invernale. Si riscontrano delle costruzioni in legno nei luoghi di passaggio, delle baite in montagna e delle cassette per lo svernamento del bestiame. Qust'ultime avevano uno scheletro in legno recintato di pali e ricoperto di paglia di segale, ma in alcuni casi erano fatte di mura di pietre. In questi luoghi si svolgevano attività industriali ed artigianali come: la lavorazione della lana, la coltivazione delle viti ecc. Molti elementi e materiali usati nella costruzione delle abitazioni nell'area di Girocastro, (come per esempio: l'uso delle lastre di pietra scolpitta o l'uso di materie miste nella costruzione dei tetti), riconfermano la tradizione remota di questo distretto nell' edificazione di abitazioni rurali.

Solitamente nella parte superiore del tetto, sopra le travi si immagazzinavano le pannocchie di mais, le patate ecc., mentre sotto la copertura del tetto si immagazzinava l'erba o la paglia usata come alimentazione invernale per il bestiame. La facciata dell'abitazione era orientata verso sudest.

Prima della Liberazione, le abitazioni rurali venivano costruite in manira libera con dei giardini circondanti fatti di cespugli o di recinzioni di mura di pietra. Le dimensioni del giardino erano proporzionate al numero dei componenti della famiglia e della loro posizione sociale. Nelle zone agricole basse ritroviamo giardini più estesi, invece nelle zone di montagna sono di dimensioni più limitate.

Foto 7. Vecchia casa, Goranxi (A. Sinani)

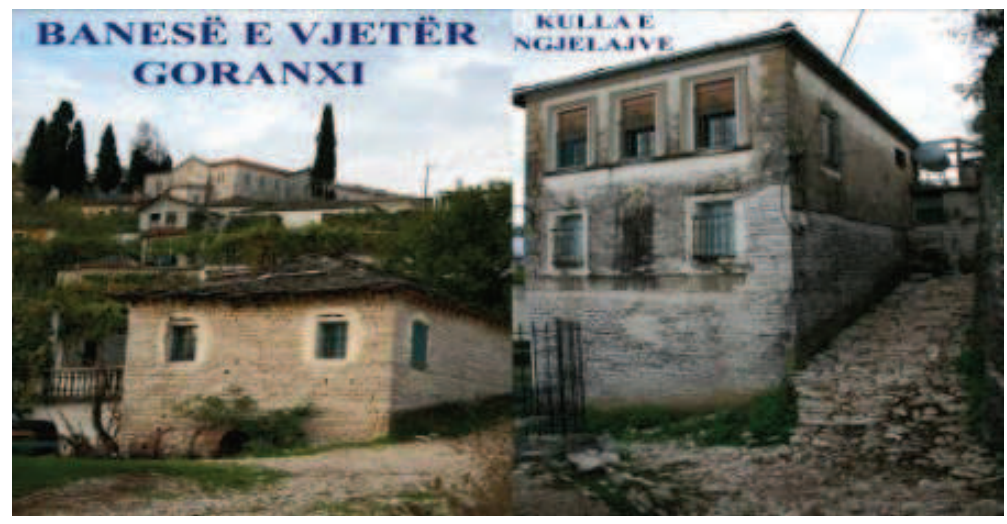

I toponimi usati sono indicativi come: di Sotto, di Sopra, Grande, Piccolo ecc. Potremmo menzionare Peshkepi di Sopra, Dropull di Sotto, Labova Grande, Labova Piccola, Labova di Sopra, Labova di Sotto ecc.

Foto 8. Vecchia casa, Labova e Zhapes (A. Sinani)

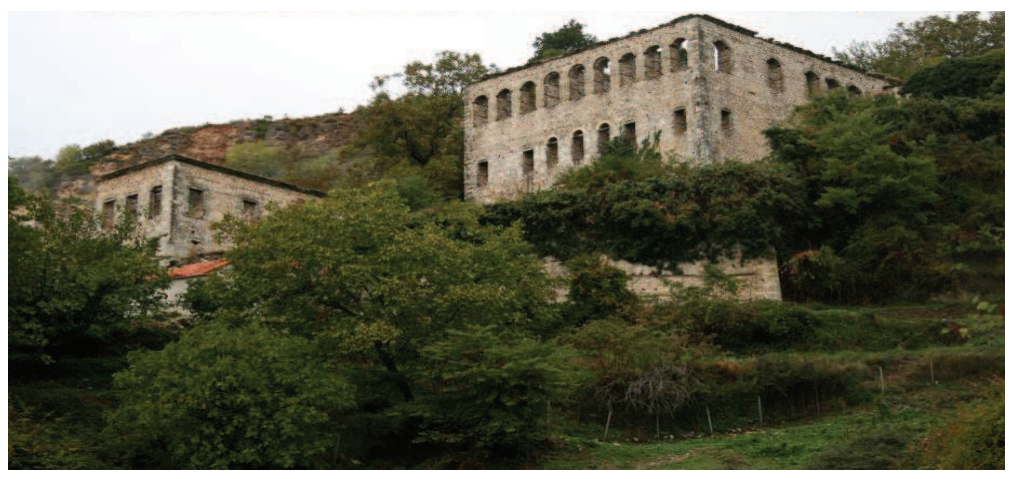




\section{Periodo $1945-1990$}

Dopo la Liberazione, nei paesini del distretto si continuò a costruire abitazioni ad uso personale, comprensivi di giardini e con una superfice di $250 \mathrm{~m}-300 \mathrm{~m}$. Le nuove abitazioni vengono innalzate all'interno delle strutture esistenti, conservando il paesaggio naturale. La continuità di tradizione nell'edificare abitazioni nei paesi del distretto è un fenomeno positivo, effettuata tramite una partecipazione diretta ed attiva degli abitanti del posto nella concezione di queste. Secondo il modello socialista sono nate nuove tradizioni edificanti come nell'area di Dropull.

Durante questo periodo sono state costruite $85.8 \%$ del fondo generale delle abitazioni nello spazio rurale. La maggior parte di stabili sono stati innalzati nei comuni di Buz (96.3\%), Luftinje (95.1\%), Frasher (94.4\%), Suke (92.9\%), Ballaban (92.1\%) e Memaliaj paese (91.9\%) del numero totale, dati questi che indicano il potanziale umano di queste aree nel periodo del socialismo ed anche la crescita della domanda d'alloggiamento della popolazione rurale.

Tab. 1. Edifici residenziali costruiti dal popolo nel periodo 1959-1971

\begin{tabular}{|lcccccc|}
\hline Anno & \multicolumn{3}{c}{1959} & & & 1965 \\
Nome & Totale & Urbano & Rurale & Totale & Urbano & Rurale \\
Paese & 7024 & 695 & 6329 & 5635 & 309 & 5326 \\
Regione di Gjirokastra & 355 & 17 & 338 & 297 & 7 & 290 \\
Gjirokastër & 171 & 9 & 162 & 94 & 3 & 91 \\
Përmet & 66 & 4 & 62 & 64 & - & 64 \\
Tepelenë & 118 & 4 & 114 & 139 & 4 & 135 \\
Anno & & 1967 & & & 1971 & \\
Nome & Totale & Urbano & Rurale & Totale & Urbano & Rurale \\
Paese & 9215 & 360 & 8855 & 6696 & 367 & 6329 \\
Regione di Gjirokastra & 609 & 20 & 589 & 673 & 143 & 530 \\
Gjirokastër & 240 & 13 & 227 & 300 & 139 & 161 \\
Përmet & 149 & 4 & 145 & 148 & - & 148 \\
Tepelenë & 220 & 3 & 217 & 225 & 4 & 221 \\
\hline
\end{tabular}

Grafico 1. Edifici residenziali costruiti dal popolo nel periodo 1959-1971

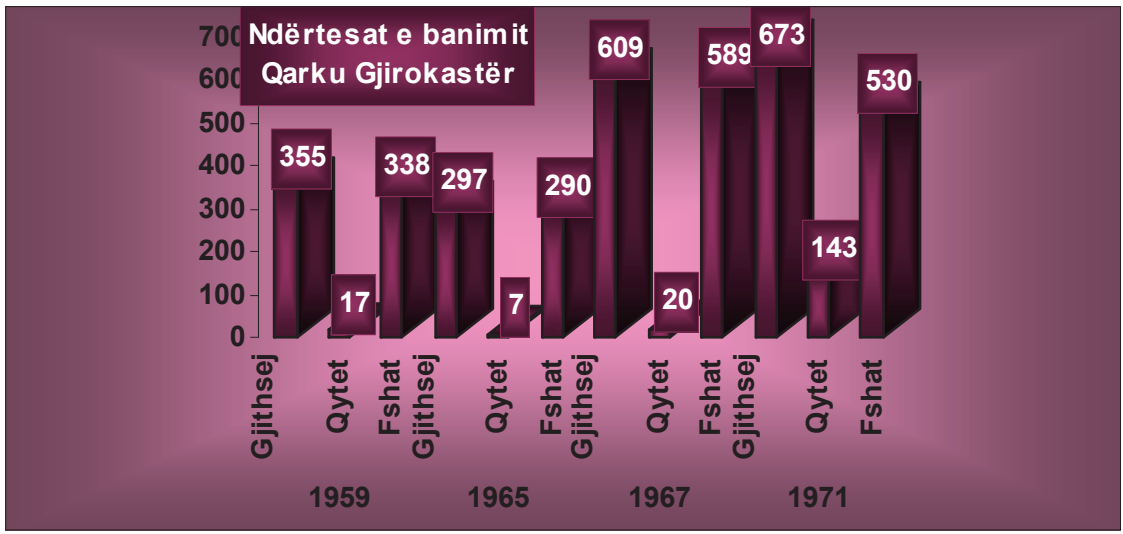




\section{Periodo 1991-2004}

Dopo l'anno 1990, le abitazioni rurali hanno subito altre trasformazioni in dipendenza ai cambiamenti dei fattori socio economici, lo stato geologico, le condizioni climatiche, il modo di vivere, l'evoluzione delle tecniche di costruzione e il cambiamento dello stile di vita. Nel paesaggio architettonico odierno dell'area rurale del distretto si possono ammirare ancora edifici appartenenti al patrimonio popolare,che si distinguono per le loro soluzioni funzionali e il loro valore architettonico.

Nelle zone di montagna gli stabili hanno un carattere più circoscritto, con finestre piccole e fatte di mura di pietre. Sono presenti anche le case di 2-3 piani che svolgono più di una funzione.

A oggi queste case sono evolute e sono state modernizzate dalle persone, che per dei periodi hanno emigrato all'estero. L'inserimento di nuovi elementi dalle abitazioni urbane aumenta con grande velocità.

Grafico 2. Immobili in fase di costruzione in zona rurale della contea Argirocastro

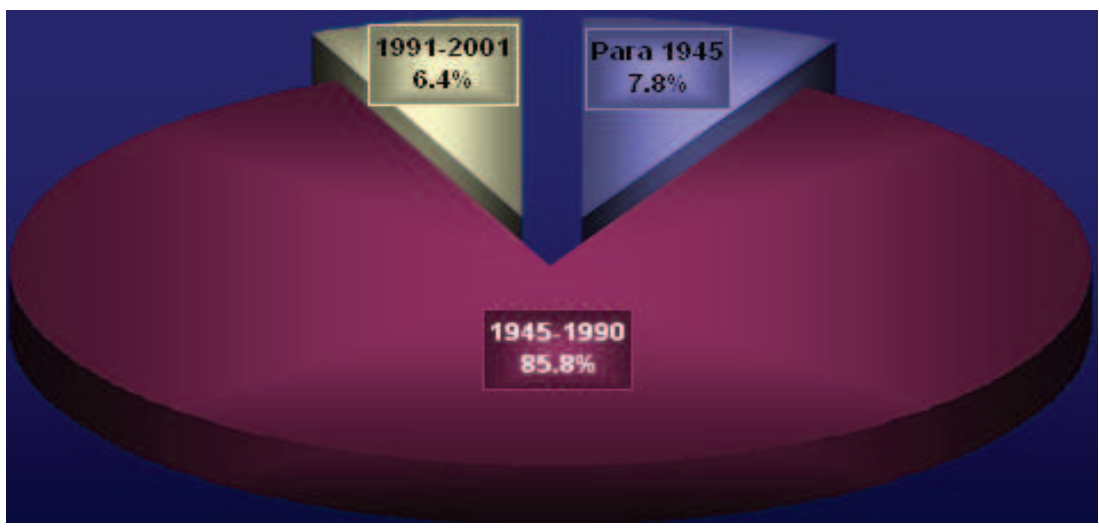

Tab. 2. Case ed edifici nel distretto di Gjirokastra secondo il censimento del 2001

\begin{tabular}{|c|c|c|c|c|c|c|c|c|c|c|}
\hline \multirow{3}{*}{$\begin{array}{c}\text { Instat } 2001 \\
\text { Abitazioni \& } \\
\text { edifici }\end{array}$} & \multicolumn{2}{|c|}{ Abitazioni totali } & \multicolumn{2}{|c|}{ Abitazioni } & \multicolumn{2}{|c|}{ Costruzione totale } & \multicolumn{3}{|c|}{ Periodo di costruzione } & \multirow{3}{*}{$\begin{array}{l}\text { Abitazioni } \\
\text { per edifici }\end{array}$} \\
\hline & & & vuote & resienziali & & & Prima 1945 & $1945-1990$ & $1991-2001$ & \\
\hline & $\mathrm{Nr}$ & $\%$ & $\%$ & $\%$ & $\mathrm{Nr}$ & $\%$ & $\%$ & $\%$ & $\%$ & \\
\hline Regione Gjirokastër & 34268 & 100 & 22 & 78 & 24136 & 100 & 11 & 80.1 & 8.9 & 1.4 \\
\hline Spazio urbane & 13705 & 40 & 16.1 & 83.9 & 4890 & 20.2 & 24 & 57.9 & 18.1 & 3 \\
\hline Spazio rurale & 20563 & 60 & 25.9 & 74.1 & 19246 & 79.8 & 7.8 & 85.8 & 6.4 & 1.1 \\
\hline Rrethi Gjirokastër & 16485 & 100 & 19.5 & 80.5 & 11680 & 100 & 16 & 73.6 & 10.4 & 1.4 \\
\hline Municipi & 7014 & 42.5 & 15.8 & 84.2 & 3019 & 25.8 & 34.3 & 50.3 & 15.4 & 2.3 \\
\hline Municipio Gjirokastër & 6364 & 38.6 & 16 & 84 & 2523 & 21.6 & 38.7 & 45.4 & 15.9 & 2.5 \\
\hline Municipio Libohovë & 650 & 3.9 & 14 & 86 & 496 & 4.2 & 11.4 & 75.6 & 13 & 1.3 \\
\hline Comuni & 9471 & 57.5 & 22.3 & 77.7 & 8661 & 74.2 & 9.6 & 81.7 & 8.7 & 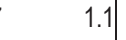 \\
\hline Antigone & 343 & 2.1 & 13.4 & 86.6 & 306 & 2.6 & 11.4 & 66 & 22.6 & 1.1 \\
\hline Cepo & 1018 & 6.2 & 28 & 72 & 996 & 8.5 & 10.7 & 83.7 & 5.6 & 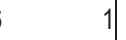 \\
\hline Dropull i Poshtëm & 1795 & 10.9 & 5.6 & 94.4 & 1625 & 13.9 & 5.4 & 85 & 9.6 & 1.1 \\
\hline Dropull i Sipërm & 2218 & 13.5 & 13.4 & 86.6 & 2120 & 18.2 & 4.2 & 88.2 & 7.6 & \\
\hline Lazarat & 683 & 4.1 & 8.9 & 91.1 & 657 & 5.6 & 7.4 & 68.9 & 23.7 & 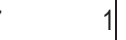 \\
\hline Lunxhëri & 977 & 5.9 & 37.3 & 62.7 & 759 & 6.5 & 24.3 & 69 & 6.7 & 1.3 \\
\hline Odrie & 273 & 1.7 & 39.6 & 60.4 & 269 & 2.3 & 12.6 & 79.2 & 8.2 & ? \\
\hline
\end{tabular}




\begin{tabular}{|c|c|c|c|c|c|c|c|c|c|c|}
\hline Picar & 651 & 3.9 & 39.3 & 60.7 & 622 & 5.3 & 7.2 & 90.2 & 2.6 & 1 \\
\hline Pogon & 556 & 3.4 & 50.5 & 49.5 & 506 & 4.4 & 10.9 & 85.6 & 3.5 & 1.1 \\
\hline Qendër Libohovë & 624 & 3.8 & 30.9 & 69.1 & 473 & 4.1 & 10 & 79.4 & 10.6 & 1.3 \\
\hline Zagorie & 333 & 2 & 36.3 & 63.7 & 328 & 2.8 & 30.1 & 69.2 & 0.7 & 1 \\
\hline Rrethi Përmet & 8413 & 100 & 26.5 & 73.5 & 6064 & 100 & 7.6 & 84.2 & 8.2 & 1.4 \\
\hline Municipi & 3292 & 39.1 & 15.8 & 84.2 & 1236 & 20.4 & 10 & 67.4 & 22.6 & 2.7 \\
\hline Municipio Përmet & 2286 & 27.2 & 14.8 & 85.2 & 614 & 10.1 & 12.1 & 58.9 & 29 & 3.7 \\
\hline Municipio Këlcyrë & 1006 & 11.9 & 18 & 82 & 622 & 10.3 & 8.1 & 75.8 & 16.1 & 1.6 \\
\hline Comuni & 5121 & 60.9 & 33.4 & 66.6 & 4828 & 79.6 & 7 & 88.5 & 4.5 & 1.1 \\
\hline Ballaban & 995 & 11.8 & 50.3 & 49.7 & 966 & 15.9 & 5.1 & 92.1 & 2.8 & 1 \\
\hline Çarçovë & 573 & 6.8 & 25.1 & 74.9 & 527 & 8.7 & 6.1 & 83.6 & 10.3 & 1.1 \\
\hline Dëshnicë & 777 & 9.2 & 36.3 & 63.7 & 757 & 12.5 & 7.1 & 90.9 & 2 & 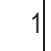 \\
\hline Frashër & 441 & 5.3 & 58.5 & 41.5 & 395 & 6.5 & 5.3 & 94.4 & 0.3 & 1.1 \\
\hline Petran & 567 & 6.8 & 6.3 & 93.7 & 524 & 8.7 & 11.9 & 75.2 & 12.9 & 1.1 \\
\hline Qendër Piskovë & 824 & 9.8 & 21.1 & 78.9 & 748 & 12.3 & 11.8 & 85.5 & 2.7 & 1.1 \\
\hline Sukë & 944 & 11.2 & 33.7 & 66.3 & 911 & 15 & 3.6 & 92.9 & 3.5 & 1 \\
\hline Rrethi Tepelenë & 9370 & 100 & 22.1 & 77.9 & 6392 & 100 & 5.2 & 88.2 & 6.6 & 1.5 \\
\hline Municipi & 3399 & 36.3 & 17 & 83 & 635 & 9.9 & 1.8 & 75.7 & 22.5 & 5.4 \\
\hline Municipio Tepelenë & 1789 & 19.1 & 10.6 & 89.4 & 429 & 6.7 & 2.6 & 70.3 & 27.1 & 4.2 \\
\hline Municipio Memaliaj & 1610 & 17.2 & 24.2 & 75.8 & 206 & 3.2 & - & 87.3 & 12.7 & 7.8 \\
\hline Comuni & 5971 & 63.7 & 25.1 & 74.9 & 5757 & 90.1 & 5.6 & 89.5 & 4.9 & 1 \\
\hline Buz & 573 & 6.1 & 44.5 & 55.5 & 571 & 8.9 & 2.8 & 96.3 & 0.9 & 1 \\
\hline Krahës & 880 & 9.4 & 13 & 87 & 854 & 13.3 & 10 & 84.3 & 5.7 & 1 \\
\hline Kurvelesh & 397 & 4.3 & 22.4 & 77.6 & 383 & 6 & 11.8 & 87.4 & 0.8 & 1 \\
\hline Lopës & 310 & 3.3 & 18.7 & 81.3 & 303 & 4.8 & 18.5 & 80.2 & 1.3 & 1 \\
\hline Luftinjë & 1190 & 12.7 & 30.8 & 69.2 & 1156 & 18.1 & 2 & 95.1 & 2.9 & 1 \\
\hline Memaliaj Fshat & 704 & 7.5 & 21.7 & 78.3 & 681 & 10.7 & 1.3 & 91.9 & 6.8 & 1 \\
\hline Qendër Tepelenë & 1259 & 13.4 & 23.5 & 76.5 & 1202 & 18.8 & 6.4 & 86.6 & 7 & 1 \\
\hline Qesarat & 658 & 7 & 25.1 & 74.9 & 607 & 9.5 & 2 & 88.6 & 9.4 & 1.1 \\
\hline
\end{tabular}

Attualmente le abitazioni rurali sono caratterizzate dalla discordanza tra le funzioni che svolgevano un tempo e quelle che deve adempiere una moderna abitazione.

Nei comuni di Frasher, Zagorie, Kurvelesh, Buz e Lope gran parte della popolazione migra all'estero, per questo motivo queste sono le aree con minor numero di costruzioni durante il periodo di transizione. Molte case sono rimaste disabitate, ma vengono utilizzate come soluzioni abitative estive, per i weekend 0 verranno usate durante l'età pensionabile per il legame psicologico che c'è. 
Grafico 3. Edifici abitabili e inabitabile in zona rurale Distretto di Argirocastro

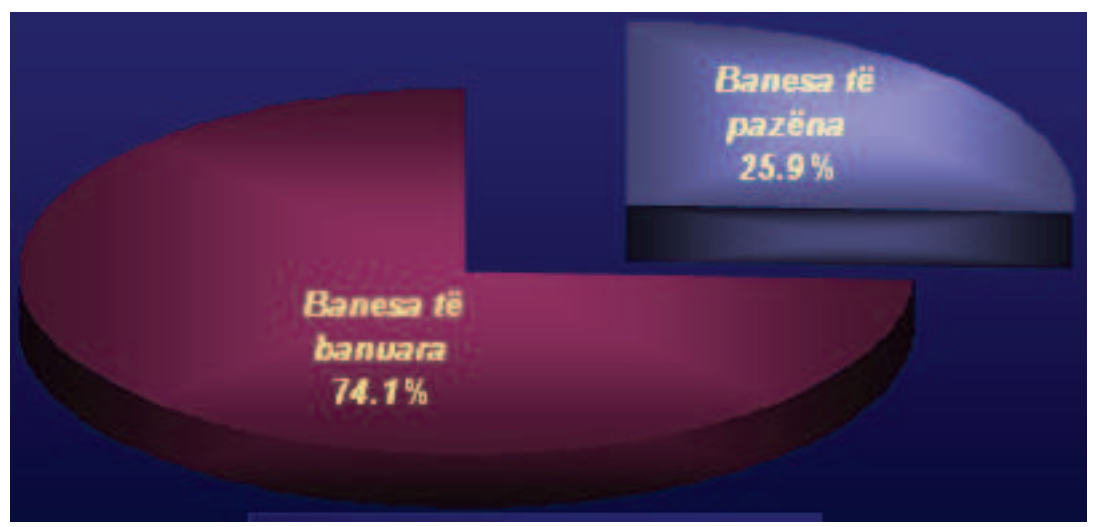

\section{Conclusions}

Lo spazio rurale del distretto di Girocastro ha ereditato abitazioni di grande valore culturale e architettonico. L'analisi di questo patrimonio unico evidenza e fa dedurre l'evoluzione delle abitazioni in diversi periodi, fa trasparire il livello della popolazione rurale,l'intensità del movimento migratorio, l'utilizzo agricolo e non del territorio, il livello di istruzione, la cultura e la qualità della vita della popolazione rurale.

Sulla base dei valori che portano, le remote costruzioni rurali devono essere:

- protette dallo Stato e le abitazioni tipiche fatte museo,

- si dovrà incrementare e difendere il loro fondo, cosi da svilupparsi il turismo storico, culturale, familiare ecc,

- si dovranno sviluppare progetti di restauro, per la manutenzione sistematica, in modo da sfruttare questa ricchezza a livello regionale, nazionale e internazionale.

\section{References}

Adhami, S. (2001). Vështrim mbi kulturën popullore të trevës së Përmetit. GERVIS, Tiranë. 22-126, 236.

Axhemi, S. (2007). Gjeografia e popullsisë dhe vendbanimeve. ALSA, Tiranë. 190-211.

Bërxholi, A. (2005). Akademia e Shkencave, Qendra e Studimeve Gjeografike, Ristrukturimi Agrar Postkomunist në

Shqipëri dhe Implikimet e Tregut të Punës, Raport Vlerësues-Studimor, Tiranë. 69.

Bërxholi, A. (1987). Ndryshimet në gjeografinë e popullsisë në zonën Vjosë-deti Jon (studim demografik), Tiranë. 9-15, 57-66.

Enciklopedia e Tepelenës, (2005). Tiranë.

Hana, L., Telo, I. (2005). Tranzicioni në Shqipëri: Arritje dhe sfida. MËSONJËTORJA, Tiranë. 185.

llirët dhe lliria nga autorët antikë. (1965). Tiranë, 133.

Lole, Dh. (2000). Përmeti dhe trevat e tij deri në vitin 1912. LUARASI, Tiranë. 14-96.

Mazniku, L. (2004). Sociologji Rurale. UBT. Tiranë. 84-90.

Misja, V., Vejsiu Y., (1987). Bërxholi A. Popullsia e Shqipërisë (Studim demografik), Tiranë. 150-163.

Muka, A. (2001). Banesa fshatare dhe familja e madhe. Botim i Akademisë së Shkencave. Tiranë. 42-75. 344-351.

Muka, A. (1990). Muret e banesës fshatare, Etnografia Shqiptare, Nr.17. Tiranë.

Riza, E. (1991). Qyteti dhe banesa shqiptare e mesjetës së vonë (shek XV-mesi i shek XIX), Akademia e Shkencave, Tiranë.

Selenica, T. (1928). Shqipria më 1927. TIRANA, Tiranë. 89.

Sheme, S. (2005). Çamëria. Vendi, popullsia dhe jeta ekonomike. "PROGRES", Tiranë. 114-117.

Shkurti, S. (1997). Etnografia Shqiptare Nr. 17. Vendbanimet fshatare dhe ekonomia bujqësore në Shqipërinë

mesjetare. Tiranë. 4-35.

Straboni. (1964). Gjeografia. Moskë. 295.

Thëngjilli, P., (2004). Historia e popullit shqiptar (395-1875), Botimet Toena, Tiranë. 268.

Tirta, M. Etnografia Shqiptare 18. Migrime të shqiptarëve. Faqe 32-76. 169-171. SHKENCA. Tiranë 1999.

Tirta, M. (1986). Lëvizje të popullsisë dhe zhvillimi i qyteteve (1945-1985), Etnografia shqiptare Nr.15, Tiranë.

Tirta, M., (1983). Vështrim rreth popullsisë së ardhur në vendbanime të krijuara pas Çlirimit në mjedise bujgësore, 
ISSN 2039-2117 (online)

ISSN 2039-9340 (print)
Mediterranean Journal of Social Sciences

Published by MCSER-CEMAS-Sapienza University of Rome
Vol 4 No 2

May 2013

Etnografia Shqiptare Nr. 13, Tiranë 1-10.

Zyrat e gjendjes civile në komunat e qarkut Gjirokastër.

Zyrat e regjistrimit të pasurive të paluajtshme në tri rrethet e qarkut Gjirokastër. 